\title{
Efficient Mobile Asset Tracking and Localization in ZigBee Wireless Network
}

\author{
R. Mardeni, Senior Member, IACSIT, and Shaifull Nizam Othman
}

\begin{abstract}
In wireless communication system, one of the unique challenges is mobile asset tracking and localization and becomes an important in the deployment of wireless enabled products. Spatial information of mobile assets is an important feature in assets management system that allows owners to gain real time insight of inventories. To meet this challenge, we developed a simple, low cost, and reliable tracking and localization system using ZibBee technology. This paper implementing a method of positioning using a Received Signal Strength Indicator (RSSI) concept. Each target asset is tagged with the blind ZigBee node and known fixed positional ZigBee nodes are used to track their positions. The RSSI values between the blind nodes and the fixed nodes are converted to the distance providing the basis for using the trilateration methods for location estimation. We also developed a software written in $\mathrm{C \#}$ that used to solve the trilateration problem and the final results of trilateration methods are mapped using Google maps. In this project, measurement and simulation were implemented and provide agreement around $89 \%$ to $92 \%$. The outcome of this study can extend current asset management functionality by providing positioning capabilities without using a positioning hardware such as the Global Positioning System (GPS), and making it suitable for a low cost deployment.
\end{abstract}

Index Terms-Mobile asset positioning, real time location system (RTLS), trilateration.

\section{INTRODUCTION}

Over the years, satellite-based Global Positioning System (GPS) has been used previously alongside the GIS applications to provide the spatial information to map real world location to the computer representation, thus making the planning and management of resources more informed. The GPS is a space-based global navigation satellite system that provides reliable location and time information in all weather conditions and at all times and anywhere on or near the Earth when there is an unobstructed line of sight to four or more GPS satellites [1]. Unfortunately, the positioning system that uses the GPS receiver adds cost to the solution infrastructure, increases power requirements to operate the on-board receiver chip and increases infrastructure maintenance points, making the deployment of such solution not commercially viable.

In this article, we proposed an alternative method using wireless sensor networks, particularly the ZigBee devices

Manuscript received May 5, 2014; revised December 10, 2014. The research is fully sponsored under Hadhari Cattle Industry Sdn Bhd (Co.No.740617-H).

R. Mardeni and Shaifull Nizam Othman are with the Faculty of Engineering, University of Multimedia, Jalan Multimedia, 63100 Cyberjaya, Selangor, Malaysia (e-mail: mardeni.roslee@mmu.edu.my, snoengineer@gmail.com). offer a more viable identification and positioning method that uses readily available facility in the ZigBee network without escalating the operational expenses. The proposed work has proven to be quite accurate, much faster and in most cases, very much cheaper than the conventional methods. The novelty of proposed ZigBee network is there has not been any existing asset identification and positioning that able to be performed simultaneously without having to invest on additional hardware for positioning. Such real time location system can be applied for tracking of mobile assets in varieties of industry such as cattle ranch, vehicle in bonded warehouse or even personnel in a large assembly plants. The main scope of our work is to do asset positioning in the ZigBee network.

\section{PROBLEM STATEMENT}

The traditional node's physical hardware and system mainly comprises of low specification and low cost component to facilitate mass production, which makes it not affordable to be deployed intensively in a monitoring zone. This has created a challenge in mapping the locations of sensor nodes as the hardware cannot provide precise timing in calculating time of flight of a packet, an important parameter in estimating distance between transmitting node and receiving node for time-based positioning methods such as Uplink-Time Difference of Arrival (U-TDOA) and Time of Arrival (TOA). The common use of cheap single Omni directional or quarter-wave monopole antenna in most of the ZigBee deployments also ruled out the possibility of using techniques that rely on packet Angle of Arrival (AoA) for estimating the location. This paper investigates positioning methodology that is based on received signal strength to estimates the distance to node and performing trilateration of those distances for localization purpose. The novelty of this proposed work is it does not require any hardware modifications to the sensor node, providing a more viable positioning methodology in ZigBee networks.

\section{WIRELESS SENSOR NETWORKS}

Wireless networks uses radio microwave to communicate with each other and has big potential in ZigBee network, it uses the $2.4 \mathrm{GHz}$ radio frequency that can be measured for positioning purposes. The ZigBee network infrastructure provides radio signal properties as part of the Quality of Service such as the Time of Arrival (ToA), Time Difference of Arrival (TDoA), Angle of Arrival (AoA) and the Received Signal Strength Indicator (RSSI) [1]. 


\section{A. Received Signal Strength Indicator (RSSI)}

It is possible to use the simple RSSI-based location algorithm if coarse accuracy is acceptable. The received signal strength can be measured for every packet received, the value indicating the signal strength is provided as part of the Link Quality Indicator (LQI) as a RSSI value and available at the PHY layer in IEEE 802.15.4 network. Using the RSSI value, a distance to node can be measured and trilateration calculation can be performed against other nodes with known positions.

\section{B. Uplink-Time Difference of Arrival (U-TDOA)}

The signal time difference received by the device from cells antenna tower is being calculated in determining the positioning of the device. Using the time difference information gathered, the distance, on the other hand, from cells antenna tower or base station to the device able to be estimated, thus leading to the coordinate positioning of the device.

\section{Time of Arrival (TOA)}

Another potential is TOA method where it is related to the U-TDOA; however, the only difference is that it uses the absolute time of arrival at a cells antenna tower or base station rather than the difference between two stations. As a result the distance can be calculated from the time of arrival as signals travel with a known velocity which in this case is the speed of light (300,000 kilometers per second). The different data of time arrival from two cells antenna tower or base station will formulate a position to two circles and the third cells antenna tower or base station is required to determine the precise device position.

\section{Angle of Arrival (AoA)}

The AoA in ZigBee networks can be achieved by grouping together three or four nodes involved in a typical radio interferometric to form an antenna array, which acts as an anchor node. The bearing of the target node can then be estimated by computing the angle of hyperbola asymptote [2]. In this method, the AoA mechanism locates the node at the point where the lines along the angles from each cells antenna intersect.

\section{Positioning Using Proposed Zigbee System IN SIMULATION}

In this work, the positioning of node using the RSSI value provided by LQI of the ZigBee node offers possibility of providing spatial data without any additional hardware requirements to the existing solution. The proposed localization process involves the use of trilateration calculation for intersection of three spheres of which the radius is obtained from the distance estimated from the RSSI value; to work this model requires that the transmitting node must be inside the intersection of three other receiver nodes of which the locations are known. One possibility to acquire a distance is measuring the received signal strength of the incoming radio signal. The idea behind RSSI is that the configured transmission power at the transmitting device $\left(P_{T X}\right)$ directly affects the receiving power at the receiving device
$\left(P_{R X}\right)$. The receiving power for device $\left(P_{R X}\right)$, can be shown as in Eq. (1).

$$
P_{R X}=P_{T X} \times G_{T X} G_{R X}(\lambda / 4 \pi d)^{2}
$$

where, $P_{R X}=$ Transmission power of sender;

$P_{T X}=$ Remaining power of wave at receiver;

$G_{T X}=$ Gain of transmitter;

$G_{R X}=$ Gain of receiver;

$\lambda=$ Wave length;

$d=$ Distance between sender and receiver.

According to Friis' free space transmission equation [3], the detected signal strength decreases quadratically with the distance to the sender. In embedded devices, the received signal strength is converted to a received signal strength indicator (RSSI) which is defined as ratio of the received power to the reference power $\left(P_{r e f}\right)$ [3]. Typically, the reference power represents an absolute value of $P_{\text {ref }}=1 \mathrm{~mW}$. The RSSI formula can be shown as in Eq. (2) as below;

$$
R S S I=10 \log \left(P_{R X} / P_{r e f}\right)
$$

An increasing received power results a rising RSSI. Distance $(d)$, is inverse proportional to RSSI. In practical scenarios, the ideal distribution of $P_{R X}$ is not applicable, because the propagation of the radio signal is interfered with a lot of influencing effects. The RSSI value is provided by the PHY layer of the ZigBee network. There are few factors that degrade and impact the RSSI values in the wireless networks and ZigBee in particular:

- Reflections on metallic objects

- Superposition of electro-magnetic fields

- Diffraction at edges

- Refraction by media with different propagation velocity

- Polarisation of electro-magnetic fields

- Unadapted MAC protocols

The RSSI based distance estimation of the target node can then be used for positioning calculation using the trilateration or multilateration formula. Few multilateration methods has been proposed to solve the localization problem in 3D space, such as Semidefinite Programming [4], MDS-MAP [5] for centralised algorithm approach and Diffusion based Multilateration [6] and Gradient based Multilateration [7]. Unfortunately, the drawbacks are found in term of cost, complexity and accuracy.

\section{A. The Mathematics of Trilateration}

Trilateration is a method of determining the relative position of objects using the geometry of triangles in a similar fashion as triangulation. Unlike triangulation, which uses angle measurements (together with at least one known distance) to calculate the subject's location, trilateration uses the known locations of two or more reference points, and the measured distance between the subject and each reference point. To accurately and uniquely determine the relative location of a point on a 2D plane using trilateration alone, generally at least three reference points are needed (at least 4 points are needed in the 3D plane). Consider the basic formula for a sphere as shown as in Eq. (3); 


$$
d^{2}=x^{2}+y^{2}+z^{2}
$$

For a sphere centred at a point $\left(x_{a}, y_{a}, z_{a}\right)$ the equation is simplified as shown as in Eq. (4);

$$
d^{2}=\left(x-x_{a}\right)^{2}+\left(y-y_{a}\right)^{2}+\left(z-z_{a}\right)^{2}
$$

Since we assume all the nodes spans out on the same plane, consider the three reference nodes $(a, b$, and $c)$ that has distance $\left(d_{a}, d_{b}, d_{c}\right)$ to the target node as in Fig. 1.

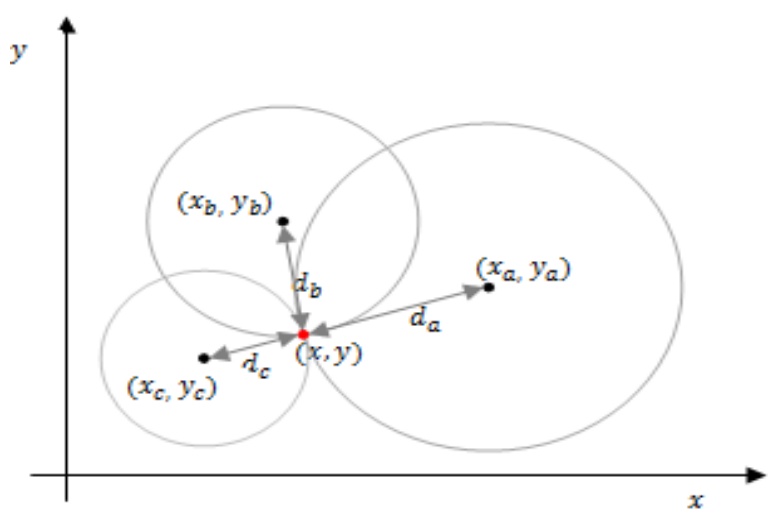

Fig. 1. Intersection of three Spheres.

The formula for the all spheres on one plane (circles) is as shown below Eq. (5), Eq. (6) and Eq. (7);

$$
\begin{aligned}
& \text { Sphere A; } d_{a}^{2}=\left(x-x_{a}\right)^{2}+\left(y-y_{a}\right)^{2} \\
& \text { Sphere B; } d_{b}^{2}=\left(x-x_{b}\right)^{2}+\left(y-y_{b}\right)^{2} \\
& \text { Sphere C; } d_{c}^{2}=\left(x-x_{c}\right)^{2}+\left(y-y_{c}\right)^{2}
\end{aligned}
$$

The Eq. (5), Eq. (6) and Eq. (7) are further expanded to become the following Eq. (8), Eq. (9) and Eq. (10);

$$
\begin{aligned}
& d_{c}^{2}=x^{2}-2 x \cdot x_{a}+x_{a}^{2}+y^{2}-2 y \cdot y_{a}+y_{a}^{2} \\
& d_{b}^{2}=x^{2}-2 x \cdot x_{b}+x_{b}^{2}+y^{2}-2 y \cdot y_{b}+y_{b}^{2} \\
& d_{c}^{2}=x^{2}-2 x \cdot x_{c}+x_{c}{ }^{2}+y^{2}-2 y \cdot y_{c}+y_{c}{ }^{2}
\end{aligned}
$$

The three Eq. (8), Eq. (9), and Eq. (10) are independent non-linear simultaneous equations which cannot be solved mathematically; however, using method proposed by Dixon [8] to obtain radical plane for sphere intersection, subtracts 2 . spheres; Eq. (10) from Eq. (9) we get the following linear Eq. (11);

$$
d_{b}{ }^{2}-d_{c}^{2}=2 x\left(x_{c}-x_{b}\right)+x_{b}{ }^{2}-x_{c}{ }^{2}+2 y\left(y_{c}+y_{b}\right)+y_{b}{ }^{2}-y_{c}{ }^{2}
$$

And subtract Eq. (8) from Eq. (9), we get the following linear Eq. (12);

$$
d_{b}^{2}-d_{a}^{2}=2 x\left(x_{a}-x_{b}\right)+x_{b}^{2}-x_{a}^{2}+2 y\left(y_{a}+y_{b}\right)+y_{b}{ }^{2}-y_{a}^{2}
$$

Rearranging the Eq. (11), to produce a variable called $V_{a}$, as Eq. (13) as follows,

$$
x\left(x_{b}-x_{c}\right)-y\left(y_{b}-y_{c}\right)=\left(x_{c}{ }^{2}-x_{b}{ }^{2}\right)+\left(y_{c}{ }^{2}-y_{b}{ }^{2}\right)+\left(d_{b}{ }^{2}-x_{b}{ }^{2}\right) / z=V_{a}
$$

Rearranging the Eq. (12), to produce a variable called $V_{b}$, as Eq. (14) as follows,

$$
x\left(x_{b}-x_{a}\right)-y\left(y_{b}-y_{a}\right)=\left(x_{a}^{2}-x_{b}^{2}\right)+\left(y_{a}^{2}-y_{b}^{2}\right)+\left(d_{b}^{2}-x_{a}^{2}\right) / z=V_{b}
$$

Resolve the Eq. (13) and Eq. (14) to gain the intersection point ' $x$ ' and ' $y$ ' of these two equations as the following Eq. (15) for ' $y$ ' value and Eq. (16) for ' $x$ ' value;

$$
\begin{gathered}
y=\frac{v_{b}\left(x_{b}-x_{c}\right)-v_{a}\left(x_{b}-x_{a}\right)}{\left(y_{a}-y_{b}\right)\left(x_{b}-x_{c}\right)-\left(y_{c}-y_{b}\right)\left(x_{b}-x_{c}\right)} \\
x=\frac{y\left(y_{a}-y_{b}\right)-v_{b}}{\left(x_{b}-x_{c}\right)}
\end{gathered}
$$

Beside, the accuracy of the localization of new system is then verified by calculating its error percentage [4]:

$$
\text { Error }(\%)=\frac{\text { estimated }- \text { actual }}{\text { actual }} \times 100
$$

\section{B. Tag Positioning Using Trilateration from Known Fixed Node Location in Field Matrix}

Trilateration (or multi lateration for that matter) in 2D surface requires intersection of at least three circles of which the coordinates of actual location is known. The real world object placement on the earth surface can be represented by few coordinates system, such as the (Latitude, Longitude pair), the Universal Transverse Mercator (UTM) and Universal Polar Stereographic (UPS) or even the crude Cartesian coordinate that place the centre of the earth as the point of origin. The latitude and longitude pair value is the most used coordinate system for civilian use, and quite simply becomes a standard for GPS and GIS mapping system.

Latitude (abbreviation: Lat., $\varphi$, or phi) is the angle from a point on the Earth's surface to the equatorial plane, measured from the center of the sphere. Lines joining points of the same latitude are called parallels, which trace concentric circles on the surface of the Earth, parallel to the equator. The North Pole is $90^{\circ} \mathrm{N}$; the South Pole is $90^{\circ} \mathrm{S}$. The $0^{\circ}$ parallel of latitude is designated the equator, the fundamental plane of all geographic coordinate systems. The equator divides the globe into Northern and Southern Hemispheres [8]. Longitude (abbreviation: Long., $\lambda$, or lambda) is the angle east or west of a reference meridian between the two geographical poles to another meridian that passes through an arbitrary point. All meridians are halves of great circles, and are not parallel. They converge at the north and south poles [9].

A fixed node is the node in the ZigBee network of which their actual position in the earth surface is known. To achieve this, each of the fixed nodes will be placed on the field alongside with the mapping tool such as the portable GPS reader and the location profile is recorded and stored in the node location registry (database). The profiling process captures locations, descriptions, device functionality and among other things the device installation history. Another solution would be to provide the GPS module on the fixed node and read the value from the device real time; this allows dynamic positioning of the fixed node and allows position 
modification to be done without manual re calibrating. The automated profiling is an ideal solution for large breeder farms or farms that does not have permanent grazing fields.

The positioning of the Fixed Node is also crucial to enable maximum field coverage and ensure that the blind node transmission signal can be read by at least three fixed nodes. To ensure optimal read coverage, the fixed node will be placed on the field using the triangular matrix configuration as in Fig. 2.
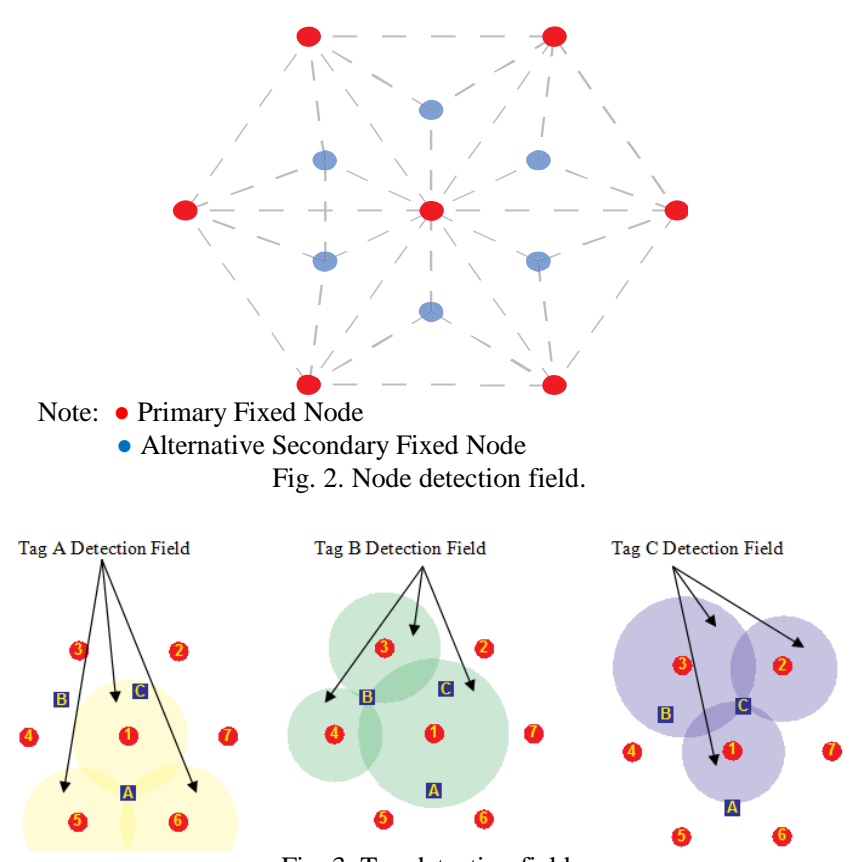

Fig. 3. Tag detection field.

where:

Maximum distance between nodes is 100 meters.

$\mathrm{A}=$ intersection point for node 1,5 and 6

$\mathrm{B}=$ intersection point for node 1,2 and 7

$\mathrm{C}=$ intersection point for node 1,3 and 4

The matrix configuration field of the fixed nodes as in Fig. 3 above will guarantee that any node that is inside the field will be detected by at least 3 fixed nodes. To improve the detection and location positioning, the alternative secondary Fixed Node can be installed as in Fig. 3.

The fixed nodes that read the blind node will log the blind node device id (MAC address), RSSI, fixed node device id and the timestamp in the log file and stored in the gateway; it is updated every two seconds.

\section{MEASUREMENT METHOD}

\section{A. Fixed Nodes Profiles}

In this project, the profiling is done manually by using a portable APOS Bluetooth GPS reader connected to a notebook running Google Earth on Microsoft Windows Vista. The fixed nodes are installed in the topology illustrated in the Fig. 3 above and each of the node location is read and stored in the "CollectorProfile" database table as well as collector location values as shown in Table I below.

The "Latitude" and "Longitude" values are stored in the decimal degree format. The "TransmitPower" is the internal settings of the node that determine the coverage radius and is not used in this demonstration. The node profile provides the fixed node positions that will be used when performing the trilateration.

TABle I: “Collector Profile” TABle Storing The Location of Fixed NODE

\begin{tabular}{|c|c|c|c|c|c|}
\hline ID & $\begin{array}{c}\text { Collector } \\
\text { Name }\end{array}$ & $\begin{array}{c}\text { Locati } \\
\text { on }\end{array}$ & $\begin{array}{c}\text { Transmit } \\
\text { Power }\end{array}$ & Latitude & Longitude \\
\hline & & & & & \\
\hline 1 & Collector1 & SFKD & 10 & 3.159167 & 101.557500 \\
\hline 2 & Collector2 & SFKD & 10 & 3.159961 & 101.558053 \\
\hline 3 & Collector3 & SFKD & 10 & 3.159997 & 101.557217 \\
\hline 4 & Collector4 & SFKD & 10 & 3.159214 & 101.556731 \\
\hline 5 & Collector5 & SFKD & 10 & 3.158542 & 101.557097 \\
\hline 6 & Collector6 & SFKD & 10 & 3.158425 & 101.557911 \\
\hline 7 & Collector7 & SFKD & 10 & 3.159403 & 101.558467 \\
\hline
\end{tabular}

\section{B. Tag Monitors}

The "TagMonitor" is tag monitor software running on the Gateway that records the tag and stores them in the log file. The following table lists the partial values extracted from the $\log$ file as shown in Table II below. However, due to the nature of the log file, which has long records, the values are summarised here to save space.

TABLE II: TAG LOCATION REGISTRY

\begin{tabular}{|c|c|c|c|c|}
\hline ID & CollectorID & TimeDetected & TagID & RSSI \\
\hline 1 & Collector1 & $24 / 01 / 201013: 26: 19$ & Tag1 & 70 \\
\hline 2 & Collector1 & $24 / 01 / 201013: 26: 19$ & Tag3 & 50 \\
\hline 3 & Collector1 & $24 / 01 / 201013: 26: 19$ & Tag4 & 65 \\
\hline 4 & Collector1 & $24 / 01 / 201013: 26: 19$ & Tag6 & 65 \\
\hline 5 & Collector1 & $24 / 01 / 201013: 26: 19$ & Tag7 & 70 \\
\hline 6 & Collector2 & $24 / 01 / 201013: 26: 19$ & Tag7 & 50 \\
\hline 7 & Collector3 & $24 / 01 / 201013: 26: 19$ & Tag2 & 65 \\
\hline
\end{tabular}

Table II shows a snapshot of test data that was gathered by the "BlindNodeMonitor" application written in Python language running on the Zigbee gateway, the DiGi XConnectPort X8 as seen in Fig. 4.

In a large scale enterprise system, the node location registry can be used as an asset movement history and separate module can be written and installed to perform the analysis. This information is a crucial part of the supply chain system.

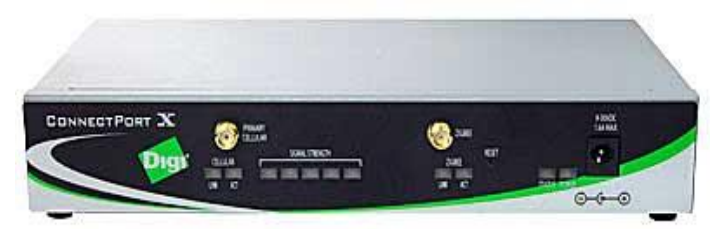

Fig. 4. DIGI international XConnectPort X8 gateway used as the fixed node.

\section{RSSI to Distance Estimation}

Received Signal Strength Indicator (RSSI) value is a useful data that is provided by the ZigBee physical layer but is not an accurate distance pointer. Due to factors discussed in the Positioning Using ZigBee Infrastructure section above, the RSSI can only provide estimation to the blind node distance from the detected fixed node. The use of distance estimation to provide localisation can generate results that are not as accurate as the results obtained from the GPS reader but can be viewed as an acceptable solution for livestock monitoring 
purposes, where exact meter to meter location is not of primary concern.

The following Table III is the field tested RSSI values achieved from the MaxStream XBee Series 2 tag node as shown in Fig. 5, communicating with DiGi XConnectPort X8 Fixed Node with clear weather and the temperature is at $31^{\circ} \mathrm{C}$. The polling exercise location is at the public park in Kota Damansara, Petaling Jaya, Selangor, Malaysia (03 ${ }^{\circ}$ 09' 33.000"N, 101 33' 27.000"E).

The distance value can be obtained using the following linear equation as shown in Eq. (18):

$$
\text { Distance }=(((-R S S I-40.0)+1.0) \times 2.0)+8.0(\text { in } m)
$$

The value illustrated in the Table III will be used throughout this project for RSSI to Distance estimation value.

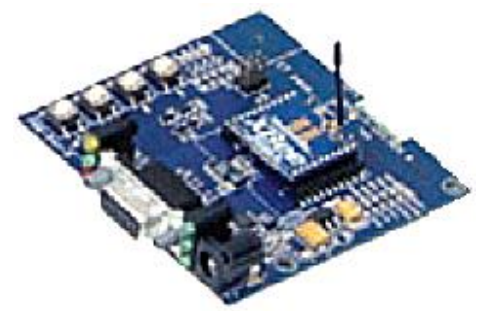

Fig. 5. DIGI international MaxStream XBee Series 2 with the development board used as the blind node.

TABLE III: RSSI TO DISTANCE TABLE

\begin{tabular}{|c|c|c|c|c|c|}
\hline $\begin{array}{l}\text { RSSI } \\
(\mathrm{dBm})\end{array}$ & $\begin{array}{l}\text { Distance } \\
(\mathrm{M})\end{array}$ & $\begin{array}{l}\text { RSSI } \\
(\mathrm{dBm})\end{array}$ & $\begin{array}{l}\text { Distance } \\
(\mathrm{M})\end{array}$ & $\begin{array}{l}\text { RSSI } \\
(\mathrm{dBm})\end{array}$ & $\begin{array}{l}\text { Distance } \\
(\mathrm{M})\end{array}$ \\
\hline-40 & 2 to 10 & -59 & 48 & -78 & 86 \\
\hline-41 & 12 & -60 & 50 & -79 & 88 \\
\hline-42 & 14 & -61 & 52 & -80 & 90 \\
\hline-43 & 16 & -62 & 54 & -81 & 92 \\
\hline-44 & 18 & -63 & 56 & -82 & 94 \\
\hline-45 & 20 & -64 & 58 & -83 & 96 \\
\hline-46 & 22 & -65 & 60 & -84 & 98 \\
\hline
\end{tabular}

\section{RESULT AND DISCUSSION}

The following section performs localization using the concept illustrated in the previous sections.

\section{A. “TagPositioning” Software Development}

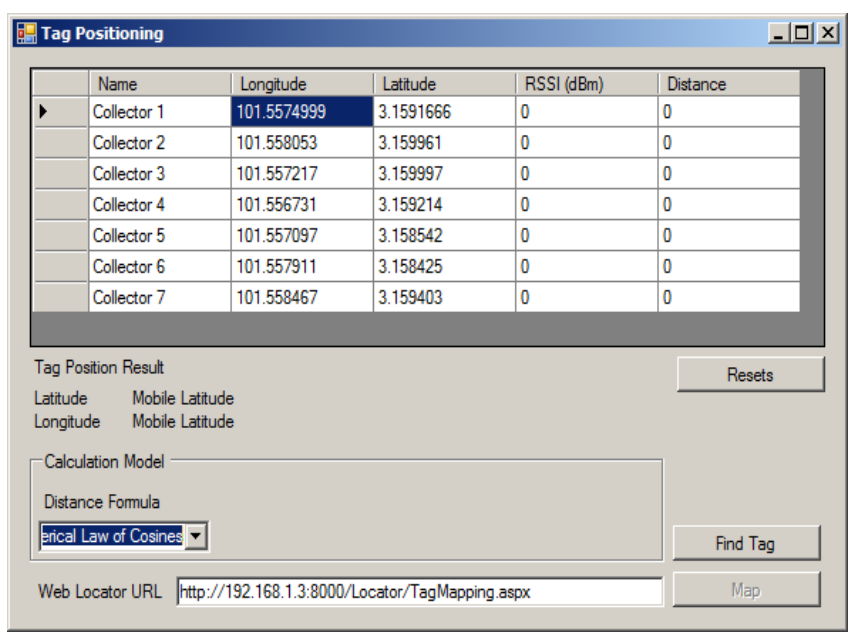

Fig. 6. TagPositioning interface.

In our work, the "TagPositioning” software developed is a
C\# application written specifically to solve the trilateration problem. The application is written using Microsoft Visual Studio and compiled on Microsoft Windows Server. The actual structure and implementation of the software are beyond the scope of this journal. At the heart of the software is a "TagPositioning" class that provides the trilateration calculation, the C\# class that implements the trilateration method, and the trilateration formula is implemented in the class as in Fig. 6.

\section{B. Solving Localisation Using "TagPositioning" Software Developed}

The software performs trilateration of blind node one at a time and maps the result using the Google Maps (http://maps.google.com) [10]. The "TagLocationRegistry" table in Table II is used for the calculation in this section. The collector position (latitude and longitude) from the "CollectorProfile" has been entered in the "TagPositioning". To find the tag position, the software needs 3 data from unique collectors. The selection of data is based on the following criteria;

1) Select all the data for Tag 1 in current minute, if data is not enough (3 unique fixed node), expand the search to include the last 5 minutes, and keep expanding for every 5 minutes until the search returns at least 10 data, if data is not found. The trilateration cannot be performed on the selected assets due to insufficient data available.

2) Sort the RSSI value in ascending order

3) Select the first 3 unique collectors that detect the Tag1.

The values from Table II are sorted and the following 3 data has been selected as shown in Table IV below;

TABLE IV: RESULT OF 3 DATA COLLECTED

\begin{tabular}{|l|l|l|l|l|}
\hline ID & CollectorID & TimeDetected & TagID & RSSI \\
\hline & & & & \\
\hline 1 & Collector1 & $24 / 01 / 201013: 26: 19$ & Tag1 & 70 \\
\hline 16 & Collector2 & $24 / 01 / 201013: 26: 20$ & Tag1 & 65 \\
\hline 17 & Collector3 & $24 / 01 / 201013: 26: 20$ & Tag1 & 55 \\
\hline
\end{tabular}

\begin{tabular}{|c|c|c|c|c|c|}
\hline \multicolumn{5}{|c|}{ 踾 Tag Positioning } & $-|\square| x$ \\
\hline & Name & Longitude & Lattude & RSSI (dBm) & Distance \\
\hline & Collector 1 & 101.5574999 & 3.1591666 & 70 & 0.07 \\
\hline & Collector 2 & 101.558053 & 3.159961 & 65 & 0.06 \\
\hline & Collector 3 & 101.557217 & 3.159997 & 55 & 0.04 \\
\hline \multirow[t]{4}{*}{ 1 } & Collector 4 & 101.556731 & 3.159214 & 0 & 0 \\
\hline & Collector 5 & 101.557097 & 3.158542 & 0 & 0 \\
\hline & Collector 6 & 101.557911 & 3.158425 & 0 & 0 \\
\hline & Collector 7 & 101.558467 & 3.159403 & 0 & 0 \\
\hline \multicolumn{5}{|c|}{ Tag Position Result } & Resets \\
\hline \multicolumn{5}{|c|}{$\begin{array}{ll}\text { Lattide } & 3^{\circ} 9^{\prime} 34.7636475923198^{\prime \prime} \\
\text { Longitude } & 101^{\circ} 33^{\prime} 26.9754302713136^{\prime \prime}\end{array}$} & \\
\hline \multicolumn{4}{|c|}{ Distance Formula } & & \\
\hline \multicolumn{5}{|c|}{ Spherical Law of $\operatorname{Cos} \nabla$} & Find Tag \\
\hline \multicolumn{5}{|c|}{ Web Locator URL hittp://Localhost:8000/Locator/TagMapping.aspx } & Map \\
\hline
\end{tabular}

Fig. 7. RSSI value gathered in Tagpositioning interface.

Finally, the final results are successfully mapped on Google Earth [10], as shown in Fig. 8, the actual locations of the tags are also recorded during the test and the results are compared (Shown in Table V). 
TABLE V: TRILATERATION RESULT

\begin{tabular}{|c|c|c|c|c|}
\hline Tag & Collector & RSSI & Tag Latitude & Tag Longitude \\
\hline \multirow[t]{3}{*}{ Tag1 } & Collector1 & 70 & 3.159657 & 101.557493 \\
\hline & Collector2 & 65 & & \\
\hline & Collector3 & 55 & & \\
\hline \multirow[t]{3}{*}{ Tag2 } & Collector4 & 50 & 3.159559 & 101.556886 \\
\hline & Collector1 & 75 & & \\
\hline & Collector3 & 75 & & \\
\hline \multirow[t]{3}{*}{ Tag3 } & Collector1 & 50 & 3.159078 & 101.557307 \\
\hline & Collector4 & 70 & & \\
\hline & Collector5 & 70 & & \\
\hline \multirow[t]{3}{*}{ Tag4 } & Collector1 & 65 & 3.158564 & 101.557419 \\
\hline & Collector5 & 55 & & \\
\hline & Collector6 & 60 & & \\
\hline \multirow[t]{3}{*}{ Tag5 } & Collector1 & 70 & 3.158722 & 101.557861 \\
\hline & Collector6 & 55 & & \\
\hline & Collector7 & 80 & & \\
\hline \multirow[t]{3}{*}{ Tag6 } & Collector1 & 65 & 3.159142 & 101.558091 \\
\hline & Collector6 & 80 & & \\
\hline & Collector7 & 55 & & \\
\hline \multirow[t]{3}{*}{ Tag7 } & Collector 1 & 70 & 3.159667 & 101.558103 \\
\hline & Collector2 & 50 & & \\
\hline & Collector7 & 60 & & \\
\hline
\end{tabular}
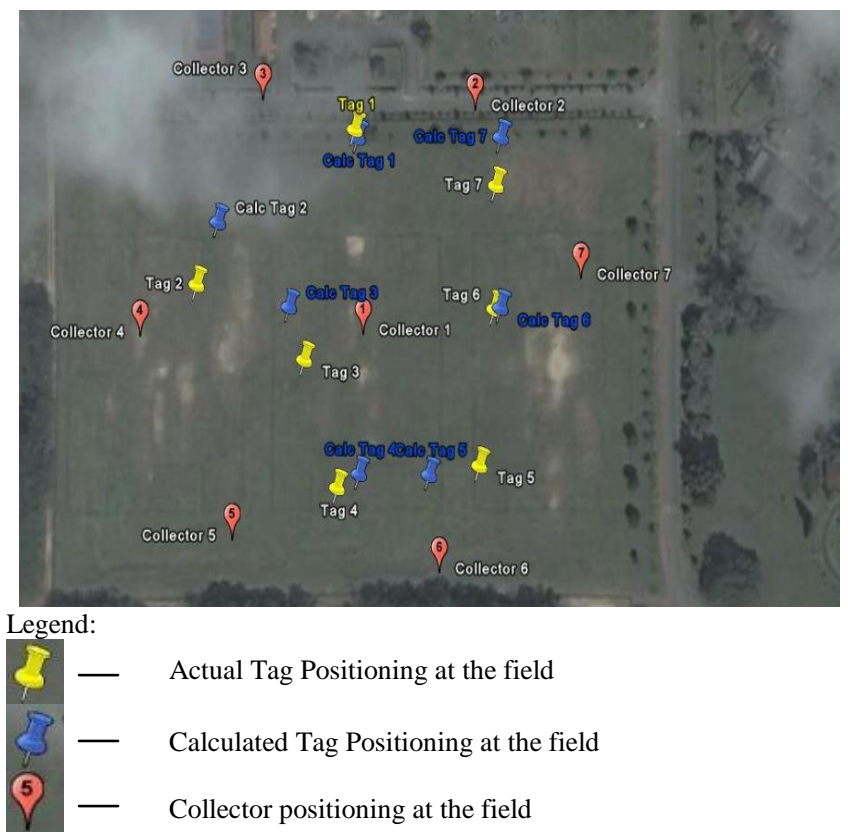

Fig. 8. Localization and tracking position result.

By using Eq. (18), the comparison has been made between actual (measured) and calculated (theory) and provide $8-11 \%$ error and agreement around $89 \%$ to $92 \%$. The percentage is significant as agreed by [4]-[7]. In comparison of traditional tracking and localization method, the proposed system will result in around USD 0.10 cost reduction of the module. In mass production this can generate significant additional revenue.

\section{CONCLUSION}

This paper implementing novel node localization in ZigBee network using new infrastructure and centralized algorithm. The software has been developed and application can be built on top of the existing solution. This will provide an inexpensive positioning solution and add functionality to the wireless sensor network applications. Further implementation of the positioning application can be built using Service Oriented Architecture (SOA) based application model which offers the flexibility of providing the location based service to an enterprise application without tearing existing solution. Finally, agreement around $89 \%$ to $92 \%$ between measurement and simulation are achieved, which is significant in wireless communication system.

\section{ACKNOWLEDGMENT}

The authors wish to express their sincere thanks to Hadhari Cattle Industry Sdn Bhd for providing the fund in this project.

\section{REFERENCES}

[1] M. Robin, Ubiquitous Positioning, Norwood, Artech House, 2008.

[2] I. Admundson, J. Sallai, X. Ketsoukos, and A. Ledeczi, "Radio interferometric angle of arrival estimation," in Proc. Wireless Sensor Networks: 7th European Conference, Portugal: Springer, 2010, p. 1.

[3] T. S. Rappaport, Wireless Communications: Principles and Practice, New Jersey, Prentice-Hall Inc., 1996.

[4] L. Doherty, P. S. Kristofer, and E. G. Laurent, "Convex position estimation in wireless sensor network," in Proc. IEEE INFOCOM 2001: the Conference on Computer Communications, 2001, p. 1655.

[5] Y. Shang, W. Ruml, Y. Zhang, and M. Fromherz, "Localization from mere connectivity," in Proc. the Fourth ACM International Symposium on Mobile Ad Hoc Networking and Computing (MobiHoc), Maryland, 2003, p. 210.

[6] S. Fitzpatrick and L. Meertens, Diffussion Based Localization, Private Communications, 2004.

[7] D. Niculescu and B. Nath, "Ad Hoc positioning using AOA," in Proc. of IEEE Infocom, San Francisco: IEEE, 2003.

[8] J. C. Dixon, Suspension Analysis and Computational Geometry, John Wiley \& Sons Ltd, 2009.

[9] Wikipedia. Geographic coordinate system. Wikipedia, the free $\begin{array}{lll}\text { encyclopedia.. (2006). } & \text { [Online]. Available: }\end{array}$ http://en.wikipedia.org/wiki/Geographic_coordinate_system

[10] Google Map Malaysia. [Online]. Available: http://maps.google.com.my/

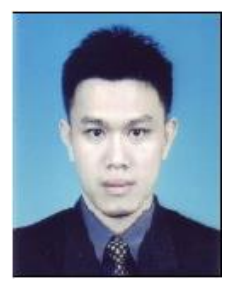

Mardeni Roslee received his bachelor degree (Hons) from UKM, master's degree in telecommunication and Ph.D. degree in communication network engineering from UPM, respectively. He is a chartered engineer awarded from UK. He has been a faculty member at the Multimedia University, Malaysia from 2008 until now. He is a senior member of IACSIT, member of IEEE and IET. He has published over 40 international journal and conference papers. He has been involved in organizing a number of conferences including APWBF09, AFICT09, PIERS12, ISTT12 and MICC13. He had guided 12 graduated postgraduate students in masters and $\mathrm{PhD}$.

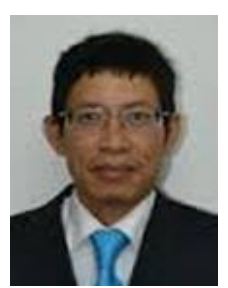

Shaifull Nizam Othamn received his bachelor degree in engineering from US and master's degree in telecommunication from Multimedia University, Malaysia. He was a senior engineer at Celcom Bhd and currently working as a manager at Celcom Bhd. He has experience working at wireless Zigbee system and worked as an engineer for many years since he joined Celcom Bhd. Apart of that, he is also active in involvement as a professional consultant for some wireless and telecommunication companies in Malaysia such as Hadhari Cattle Sdn Bhd. 